\section{Cahiers de Narratologie}

Analyse et théorie narratives

$18 \mid 2010$

Littérature et sciences

\title{
Avant-propos à la section « Littérature et Médecine »
}

\section{Edwige Comoy Fusaro}

\section{(2) OpenEdition \\ 1 Journals}

Édition électronique

URL : http://journals.openedition.org/narratologie/6057

DOI : $10.4000 /$ narratologie.6057

ISSN : 1765-307X

Éditeur

LIRCES

\section{Référence électronique}

Edwige Comoy Fusaro, "Avant-propos à la section "Littérature et Médecine » », Cahiers de Narratologie [En ligne], 18 | 2010, mis en ligne le 02 juillet 2010, consulté le 21 septembre 2020. URL : http://journals.openedition.org/narratologie/6057 ; DOI : https://doi.org/10.4000/narratologie.6057

Ce document a été généré automatiquement le 21 septembre 2020.

\section{(c) (i) () $९$}

Cahiers de Narratologie - Analyse et théorie narratives est mis à disposition selon les termes de la licence Creative Commons Attribution - Pas d'Utilisation Commerciale - Pas de Modification 4.0 International. 


\title{
Avant-propos à la section «Littérature et Médecine»
}

\author{
Edwige Comoy Fusaro
}

1 «Littérature et Médecine »: telle était l'appellation de la Journée d'étude qui s'est tenue le 10 octobre 2008 à l'Université Nice Sophia Antipolis. La médecine est sans conteste l'enfant chérie des intersections entre sciences et littérature - le radical indoeuropéen med- (prendre soin de) n'a-t-il pas donné, parallèlement à 'médecine' (du latin mederi : soigner), 'méditer' (du grec medomai: méditer) et, par suite, 'imaginer', 'inventer'? La médecine est peut-être la moins scientifique des sciences, celle qui partage le plus d'affinités avec les sciences humaines et les humanités. Cela tient notamment au caractère littéraire de la clinique : le médecin doit interpréter les signes d'un corps, singulier et unique, en les comparant aux normes recensées dans la littérature médicale sans perdre de vue l'idiosyncrasie de son patient. Un même symptôme peut être l'indice d'une multitude de maladies et une même maladie produire des symptômes différents chez différents sujets. Par ailleurs, le médecin n'échappe pas à l'influence de son contexte socio-historique, politique, idéologique, de sorte que l'on a vu de véritables modes médicales sévir durablement sans qu'aucune légitimité thérapeutique avérée ne les justifient, comme ce fut le cas des saignées. Quant à la réception et à la mémoire que la collectivité garde des évolutions des sciences médicales, Louise L. Lambrich a clairement établi que notre histoire (occidentale) de la médecine s'apparente plutôt à une «mythologie ». Il était donc tout naturel, dans le cadre du séminaire Littérature et Sciences, de consacrer aux rapports entre médecine et littérature une réflexion spécifique.

2 Ces travaux s'inscrivent dans une recherche bien plus vaste, qui a connu un nouvel essor récemment et à laquelle il convient de rendre hommage. On peut citer notamment quelques-uns des livres qui ont paru sur la question ces dernières années, sous la plume de Gérard Danou - fer de lance de ce renouveau -, de Vincent Kaufmann, Bertrand Marquer ou Louis Van Delft ${ }^{1}$, les séminaires de l'Université de CergyPontoise ${ }^{2}$, ou encore les colloques «Littérature et médecine : approches et perspectives (XVIe-XIXe siècles) » (Genève, 2005), «Médecine, sciences de la vie et littérature » 
(Grenoble, 2008), « Médecine et narration au XVIIIe siècle » (Maison française d'Oxford, 2008). Dans un contexte aussi riche, ce numéro des Cahiers de narratologie se propose d'apporter une pierre à l'édifice dans une perspective délibérément interdisciplinaire, en sollicitant des chercheurs en littérature française et étrangère, des philosophes et des civilisationnistes (d'où l'antéposition de la littérature vis-à-vis de la médecine dans l'intitulé de la Journée), avec des contributions qui portent sur les XIXe et XXe siècles. En effet, si l'association de la médecine et de la littérature fait indéniablement partie de notre patrimoine culturel immémorial - il n'est que de songer aux médecins poètes, de Nicandre de Colophon à Rabelais -, depuis l'avènement de l'ère industrielle le ménage s'est considérablement enrichi de nouvelles problématiques.

3 Les deux premières contributions s'intéressent au rôle de la médecine dans la vie sociale, dans une perspective philosophique, idéologique et politique. La médecine y est proprement l'art de prévenir et de soigner les maladies, c'est-à-dire une praxis, une " activité en vue d'un résultat », selon la définition du Robert, et la littérature est son instrument de prédilection. Frédéric Dupin étudie le statut central de la médecine chez Auguste Comte (1798-1857), dont l'ambitieuse réforme politique prévoit que seule la connaissance d'une sélection très restreinte de saines œuvres littéraires, atemporelles et universelles, comme celles de Marc Aurèle, de Dante ou de Cervantes, garantira une hygiène cérébrale indispensable à la bonne marche de la société.Alors que, à la suite de Pinel et Esquirol et sous l'impulsion décisive de Claude Bernard, la médecine prend une autorité grandissante en se proclamant "anatomique " et " expérimentale ", Comte prétend lui ôter toute spécificité dogmatique, en faire un art global qui dépasse l'étroite physio-pathologie et investisse la sociologie et la morale. Marie-Aline Barrachina se penche quant à elle sur l'œuvre complexe du docteur Gregorio Marañón (1887-1960), endocrinologue de renom, qui met son autorité scientifique au service du birth control mais que le régime franquiste instrumentalise, paradoxalement, pour servir une politique nataliste et une idéologie sexiste.

Les deux autres contributions ciblent plus précisément la fonction de la médecine au regard de la littérature fictionnelle, et vice-versa. Après avoir cerné la spécificité de la pensée d'Alexandre Brierre de Boismont (1797-1881) vis-à-vis des aliénistes esquiroliens, Jean-Louis Cabanès relève les échos de ses thèses scientifiques chez Flaubert dans la troisième version de La Tentation de saint Antoine où le «cadre épistémique » qui constitue le socle des conceptions médicales sur l'imagination - audelà des différences individuelles - offre à l'auteur le "cadre rhétorique " de son œuvre. Flaubert s'affranchit néanmoins des théories de Brierre lui-même en posant une conception originale, proto-freudienne, de l'hallucination et en revendiquant l'autonomie du processus créatif. La médecine demeure cependant une base spéculative de référence tandis que chez l'écrivain italien Camillo Boito (1836-1914), dont Edwige Comoy Fusaro analyse une nouvelle, la médecine n'est plus d'aucune utilité pour l'écrivain : le regard poétique, relayé dans le texte par la vision pathologique, pallie les défaillances manifestes de l'œil médical. Les résultats auxquels parvient Boito, de façon encore plus marquée que chez Flaubert, rejoignent ainsi ante litteram le discours à venir de Sigmund Freud (1856-1939) sur la littérature, qui devait reconnaître à certaines œuvres de remarquables qualités intuitives vis-à-vis des théories psychanalytiques postérieures. 


\section{NOTES}

1. Cfr.par exemple LOUIS VAN DELFT, Littérature et anthropologie. Nature humaine et caractère à l'âge classique, Paris, PUF, 1993 ; GÉRARD DANOU, Le corps souffrant. Littérature et médecine, Seyssel, Editions Champ Vallon, 1994 ; VINCENT KAUFMANN, Ménage à trois : littérature, médecine, religion, Villeneuve d'Asq, Presses Universitaires du Septentrion, 2007; BERTRAND MARQUER, Les "Romans" de la Salpêtrière. Réception d'une scénographie clinique: Jean-Martin Charcot dans l'imaginaire fin-de-siècle, Genève, Droz, 2008.

2. Cfr. Littérature et médecine ou les pouvoirs du récit (2001), Arts littéraires, arts cliniques (2003), Peser les mots (2007). 